\title{
Using DTSA-II to Simulate and Interpret an X-ray Spectrum Image from a K411 Microparticle
}

\author{
Nicholas W. M. Ritchie*
}

* Surface and Microanalysis Science Division, National Institute of Standards and Technology, 100 Bureau Drive; MS: 8371, Gaithersburg, MD 20882

In the real world, not all the materials on which we want to perform x-ray microanalysis are available as nice simple, flat, polished, homogeneous bulk samples. Often the material is only available in particulate form or only present as an inclusion in a matrix. Most of the quantitative algorithms we us regularly assume idealized samples in which, with the exception of backscattered electrons, the energetic probe electrons are constrained to remain within the sample material. Furthermore assumptions are made about the geometry of the material through which the x-rays must travel to reach the detector. These assumptions rarely hold for particulate or inclusion samples.

Instead other methods have been developed to address particle samples. Often the best bet is to ignore corrections for atomic number, absorption and fluorescence and simply normalize the ratio between the intensity measured on the sample and the intensity measured from elemental standards (normalized k-ratio method.) Alternatively, one may collect high count spectra and use the peak-tobackground method[1]. When the particle geometry is simple and can be measured, Armstrong's particle correction algorithm[2] may be appropriate. Regardless, quantifying particle spectra is more difficult, the tools are less refined and the results less reliable.

DTSA-II is a software tool for simulating and quantifying x-ray microanalysis data. One of the long term goals for the DTSA-II platform is to facilitate some common but difficult microanalysis measurements. Particles represent the first such problem that we are addressing. While the literature documents over thirty years of research into particulate analysis, there is little consensus on the best approach. DTSA-II is a perfect tool to assess the classic approaches and potentially develop new ones. The combination of Monte Carlo simulation tools and quantitative microanalysis tools within DTSA-II is unique and powerful.

The first necessary step is to validate that DTSA-II is capable of producing credible simulations of particle spectra. The approach we have chosen to take is to apply our high performance silicon drift detector to measure high spatial resolution, high count depth x-ray spectrum images of well characterized glass microparticles. Possibly the best characterized microanalysis-suitable glass is NIST SRM-2066 - K411 Glass Microspheres[3]. Many of these particles are highly spherical and the composition is certified. The maps in Figure 1a) were collected at $25 \mathrm{keV}$ from a $3.3 \mu \mathrm{m}$ diameter K411 sphere. At $25 \mathrm{keV}$, the ionization ranges of the transitions of interest exceed $5 \mu \mathrm{m}$ so the maps shows a wealth of particle specific phenomena. We then used the script-based Monte Carlo modeling capabilities of DTSA-II to simulate pixel-by-pixel the X-ray map $(19,200$ spectra in $\sim 10$ hours on a $2.8 \mathrm{GHz}$ Mac Pro). Figure $1 \mathrm{~b}$ shows the simulated maps. Figure 2 looks more closely at a linescan parallel to the detector axis. These plots suggest that DTSA-II is highly capable of simulating particle spectra. We will present more detailed studies in which we use the unique capabilities of Monte Carlo simulation to interpret and understand the phenomena which make particles hard to quantify and to evaluate the classic particle analysis strategies.

References

[1] J.A. Small et al., in Characterization of Particles, K.F.J. Heinrich, ed., NBS Spec. Pub. 460, 29-38 
[2] J.T. Armstrong in Electron Probe Quantification, K.F.J. Heinrich \& D.E. Newbury, eds., Plenum Press, New York, 1991, 261-315

[3] SRM-2066 Certificate - https://www-s.nist.gov/srmors/view_cert.cfm?srm=2066
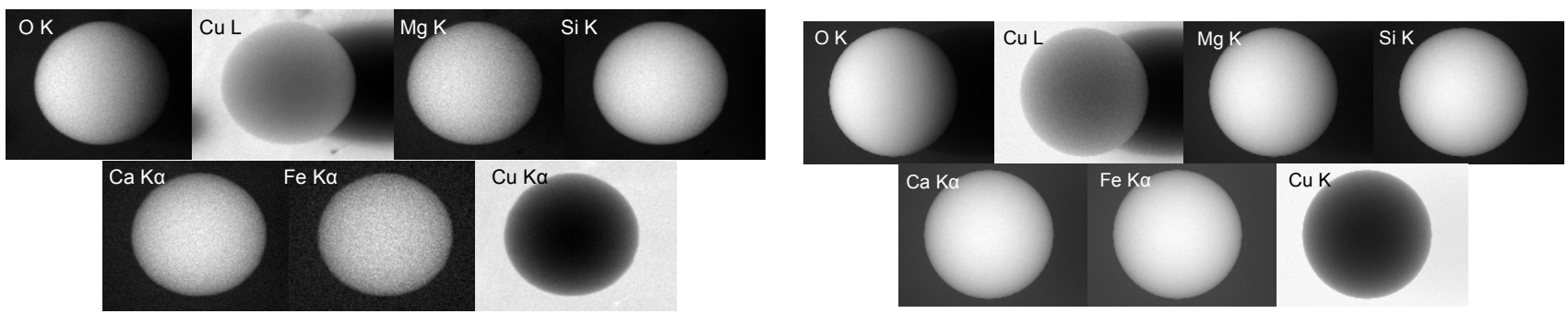

Fig.1. Compares x-ray maps collected on a silicon drift detector at $25 \mathrm{keV}$ from a $3.3 \mu \mathrm{m}$ diameter microsphere of K411 glass (a) with a DTSA-II Monte Carlo simulation of the same (b). The Monte Carlo model is seen to simulated all the important phenomena which complicate the interpretation of particle spectra. The detector is located to the left. The right edge of the O K map shows the effects of absorption. The $\mathrm{Cu} \mathrm{L}$ map shows the influence of electrons passing through the particle and of $\mathrm{x}-$ ray shadowing by the particle. The edges of the particle in the $\mathrm{Cu} \mathrm{K \alpha}$ map shows the effect of electrons scattering off the particle onto the substrate.

$\mathrm{OK}$

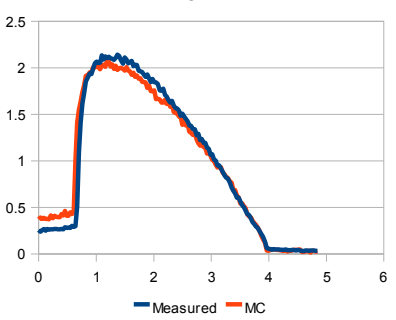

SiK
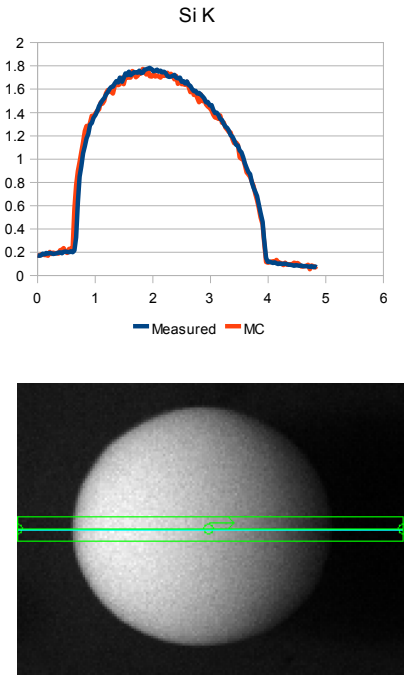

$\mathrm{CuL}$

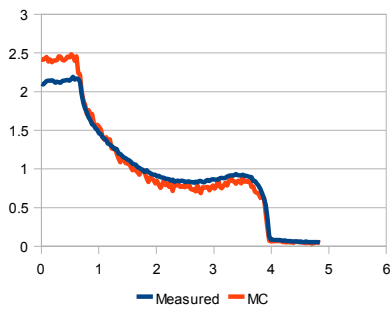

$\mathrm{CaK}$

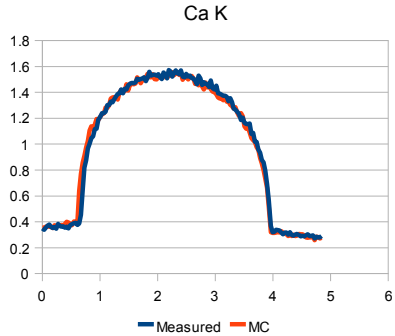

CuK

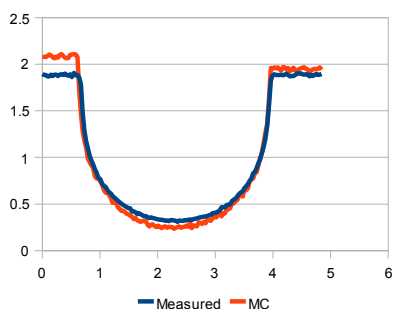

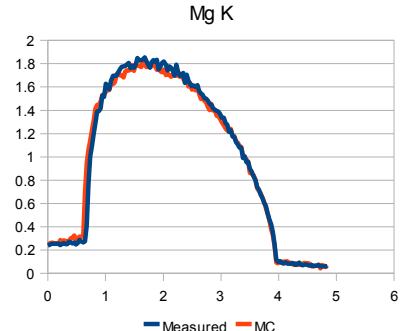
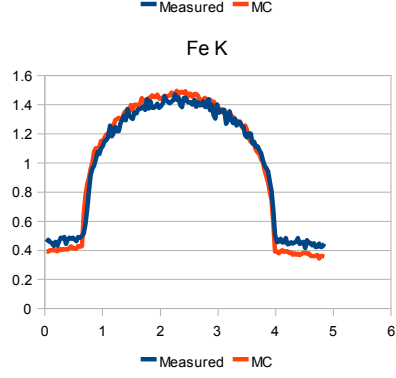

- Measured $-\mathrm{M}$

Scaled to average of all data.

Fig. 2. These plots make the general trends seen in Fig.1. more quantitative. The slice of pixels shown in the lower-left image are accumulated and plotted for the measured and simulated maps. The plots demonstrate that the model is particularly good at simulating the elements within the particle. 